\title{
Time to Diagnosis Possibly Reflects Bipolar II Disorder Heterogeneity
}

\section{Yasuko Fuse-Nagase*}

University Health Center, Ibaraki University, 2-1-1 Bunkyo, Mitoshi, Ibaraki 310-8512, Japan

"Corresponding author: Yasuko Fuse-Nagase, University Health Center, Ibaraki University, 2-1-1 Bunkyo, Mitoshi, Ibaraki, 310-8512, Japan, Tel:+81-29-228-8081; Fax: +81-29-228-8084; E-mail: yfusen@mx.ibaraki.ac.jp

Received date: Apr 1, 2014, Accepted date: Apr 26, 2014, Published date: Apr 30, 2014

Citation: Fuse-Nagase Y (2014) Time to Diagnosis Possibly Reflects Bipolar II Disorder Heterogeneity. Brain Disord Ther 3: 122. doi: 10.4172/2168-975X.1000122

Copyright: (C) 2014 Fuse-Nagase Y. This is an open-access article distributed under the terms of the Creative Commons Attribution License, which permits unrestricted use, distribution, and reproduction in any medium, provided the original author and source are credited

\section{Letter to Editor}

Bipolar II disorder (BDII) is one of the mental, neurological and substance-use disorders that constitute the global burden of disease [1]. It is a neurodevelopmental psychiatric disorder [2], but little is known about its biological mechanism. If some marker of (BDII), that is easy to detect and can predict important prognoses as to the risk of suicidal behavior [3], response to treatment [4], etc., is identified, it would be very helpful in clinical settings. Twenty outpatients visiting a psychiatric practice in Tokyo on June 1, 2012, who were diagnosed with BDII (DSM-IV-TR) [5], were investigated. Of 20 patients, 6 had been diagnosed with BDII before their first visit to the practice and excluded from further investigation. The ages of the 14 patients ( 4 men and 10 women) included in further investigation were between 43 and 77. Two of them had comorbid anxiety disorder. Based on the time from the first visit to be diagnosed with bipolar II disorder, patients were clearly divided into 2 groups, one with a diagnosis of less than 2 years (the "shorter group") that included 8 patients, and the other with a diagnosis of more than 5 years (the "longer group") that included 6 patients.. For 10 patients who had been followed-up for $>2$ years ( 4 in shorter group and 6 in longer group), the number of episodes in the last 2 years (major depressive and hypomanic) was calculated. The Mann-Whitney's U-test was performed to assess the difference in number of episodes between the 2 groups. The data were analyzed using SPSS 21.0. The patients in the longer group tended to have more episodes than those in the shorter group, although this difference was not statistically significant $(\mathrm{U}=4.50, \mathrm{~ns}$.).

All the patients visited the practice once in every 4 weeks or more frequently and there were very little chance of delay in diagnosis due to fewer visits. There was no case with comorbid substance use disorder that could have confounded diagnosis. Once being diagnosed with BDII, all the patients were continuously treated with one or two mood stabilizers, and with antipsychotics if necessary. Thus having more episodes was not due to incorrect treatment. The findings suggest that BDII is heterogeneous and that there may be a subgroup of patients with BDII who do not have hypomanic episodes in early stage but respond poorly to mood stabilizers. This is a hypothesis and further study using a larger sample size is necessary to verify it. Time to diagnosis can be a non-invasive and easy to detect marker.

Part of this study was presented at the World Psychiatric Association International Congress 2013.

\section{References}

1. Collins PY, Patel V, Joestl SS, March D, Insel TR, et al. (2011) Grand challenges in global mental health. Nature 475: 27-30.

2. Geoffroy PA, Etain B, Scott J, Henry C, Jamain S, et al. (2013) Reconsideration of bipolar disorder as a developmental disorder: importance of the time of onset. J Physiol Paris 107: 278-285.

3. Chaudhury SR, Grunebaum MF, Galfalvy HC, Burke AK, Sher L, et al. (2007) Does first episode polarity predict risk for suicide attempt in bipolar disorder? J Affect Disord 104: 245-250.

4. Calabrese JR, Fatemi SH, Kujawa M, Woyshville MJ (1996) Predictors of response to mood stabilizers. J Clin Psychopharmacol 16: 24S-31S.

5. American Psychiatric Association (2000) Diagnostic and Statistical Manual of Mental Disorders, Fourth Edition, Text Revision: DSM-IVTR. WashingtonDC. 